\title{
Convex Variational Formulation with Smooth Coupling for Multicomponent Signal Decomposition and Recovery
}

\author{
Luis M. Briceño-Arias ${ }^{1}$ and Patrick L. Combettes ${ }^{2, *}$ \\ ${ }^{1}$ UPMC Université Paris 06, Laboratoire Jacques-Louis Lions - UMR 7598 and \\ Équipe Combinatoire et Optimisation - UMR 7090, 75005 Paris, France. \\ ${ }^{2}$ UPMC Université Paris 06, Laboratoire Jacques-Louis Lions - UMR 7598, 75005 \\ Paris, France.
}

Received 5 August 2009; Accepted (in revised version) 8 August 2009

\begin{abstract}
A convex variational formulation is proposed to solve multicomponent signal processing problems in Hilbert spaces. The cost function consists of a separable term, in which each component is modeled through its own potential, and of a coupling term, in which constraints on linear transformations of the components are penalized with smooth functionals. An algorithm with guaranteed weak convergence to a solution to the problem is provided. Various multicomponent signal decomposition and recovery applications are discussed.
\end{abstract}

AMS subject classifications: 94A12, 90C25

Key words: Convex optimization, denoising, image restoration, proximal algorithm, signal decomposition, signal recovery.

\section{Problem statement}

The processing of multicomponent signals has become increasingly important due, on the one hand, to the development of new imaging modalities and sensing devices, and, on the other hand, to the introduction of sophisticated mathematical models to represent complex signals. It is for instance required in applications dealing with the recovery of multichannel signals $[8,33,34,40]$, which arise in particular in color imaging and in the multi- and hyperspectral imaging techniques used in astronomy and in satellite imaging. Another important instance of multicomponent processing is found in signal decomposition problems, e.g., $[2,5-7,15,43,44]$. In such problems, the ideal signal is viewed as a mixture of elementary components that need to be identified individually.

${ }^{*}$ Corresponding author. Email addresses: lbriceno@math.jussieu.fr (L. M. Briceño-Arias), plc@math. jussieu.fr (P. L. Combettes) 
Mathematically, a multicomponent signal can be viewed as an $m$-tuple $\left(x_{i}\right)_{1 \leq i \leq m}$, where each component $x_{i}$ lies in a real Hilbert space $\mathscr{H}_{i}$. A generic convex variational formulation for solving multicomponent signal recovery or decomposition problems is

$$
\underset{x_{1} \in \mathscr{H}_{1}, \cdots, x_{m} \in \mathscr{H}_{m}}{\operatorname{minimize}} \Phi\left(x_{1}, \cdots, x_{m}\right),
$$

where $\left.\left.\Phi: \mathscr{H}_{1} \oplus \cdots \oplus \mathscr{H}_{m} \rightarrow\right]-\infty,+\infty\right]$ is a convex cost function. At this level of generality, however, no algorithm exists to solve (1.1) reliably in the sense that it produces $m$ sequences $\left(x_{1, n}\right)_{n \in \mathbb{N}}, \cdots,\left(x_{m, n}\right)_{n \in \mathbb{N}}$ converging (weakly or strongly) to points $x_{1}, \cdots, x_{m}$, respectively, such that $\left(x_{i}\right)_{1 \leq i \leq m}$ minimizes $\Phi$. Let us recall that, even in the elementary case when $m=2$ and $\mathscr{H}_{1}=\mathscr{H}_{2}=\mathbb{R}$, the basic Gauss-Seidel alternating minimization algorithm does not possess this property [28]. In this paper, we consider the following, more structured version of (1.1).

Problem 1.1. Let $m \geq 2$ and $p \geq 1$ be integers, let $\left(\mathscr{H}_{i}\right)_{1 \leq i \leq m}$ and $\left(\mathscr{G}_{k}\right)_{1 \leq k \leq p}$ be real Hilbert spaces, and let $\left(\tau_{k}\right)_{1 \leq k \leq p}$ be in $] 0,+\infty\left[\right.$. For every $i \in\{1, \cdots, m\}$, let $\left.\left.f_{i}: \mathscr{H}_{i} \rightarrow\right]-\infty,+\infty\right]$ be a proper lower semicontinuous convex function and, for every $k \in\{1, \cdots, p\}$, let $\varphi_{k}: \mathscr{G}_{k} \rightarrow \mathbb{R}$ be convex and differentiable with a $\tau_{k}$-Lipschitz continuous gradient, and let $L_{k i}: \mathscr{H}_{i} \rightarrow \mathscr{G}_{k}$ be linear and bounded. It is assumed that $\min _{1 \leq k \leq p} \sum_{i=1}^{m}\left\|L_{k i}\right\|^{2}>0$. The problem is to

$$
\underset{x_{1} \in \mathscr{H}_{1}, \cdots, x_{m} \in \mathscr{H}_{m}}{\operatorname{minimize}} \sum_{i=1}^{m} f_{i}\left(x_{i}\right)+\sum_{k=1}^{p} \varphi_{k}\left(\sum_{i=1}^{m} L_{k i} x_{i}\right),
$$

under the assumption that solutions exist.

Let us note that (1.2) is a particular case of (1.1), in which $\Phi$ is decomposed in two terms, namely

$$
\Phi\left(x_{1}, \cdots, x_{m}\right)=\underbrace{\sum_{i=1}^{m} f_{i}\left(x_{i}\right)}_{\text {separable term }}+\underbrace{\sum_{k=1}^{p} \varphi_{k}\left(\sum_{i=1}^{m} L_{k i} x_{i}\right)}_{\text {coupling term }} .
$$

Each function $f_{i}$ in the separable term promotes an intrinsic property of the $i$ th component $x_{i}$ of the signal. On the other hand, the coupling term models $p$ interactions between the $m$ components $\left(x_{i}\right)_{1 \leq i \leq m}$. An elementary interaction is associated with a potential $\varphi_{k}$ acting on a linear transformation $\sum_{i=1}^{m} L_{k i} x_{i}$ of the components. The coupling is smooth in the sense that the function $\varphi_{k}$ is differentiable with a Lipschitz gradient. As will be seen in subsequent sections, Problem 1.1 not only captures existing formulations for which reliable solution methods are not available, but it also allows us to investigate a wide range of new problems. In addition, it can be solved reliably by the following proximal algorithm recently developed in [4] (the definition of the proximity operator $\operatorname{prox}_{f_{i}}$ of a convex function $\left.\left.f_{i}: \mathscr{H}_{i} \rightarrow\right]-\infty,+\infty\right]$ is given in Section 2.2).

Algorithm 1.1. Set

$$
\beta_{1}=\frac{1}{p \max _{1 \leq k \leq p} \tau_{k} \sum_{i=1}^{m}\left\|L_{k i}\right\|^{2}}
$$


and fix $\varepsilon$ in $] 0, \min \left\{1, \beta_{1}\right\}\left[,\left(\lambda_{n}\right)_{n \in \mathbb{N}}\right.$ in $[\varepsilon, 1],\left(\gamma_{n}\right)_{n \in \mathbb{N}}$ in $\left[\varepsilon, 2 \beta_{1}-\varepsilon\right]$, and $\left(x_{i, 0}\right)_{1 \leq i \leq m}$ in $\mathscr{H}_{1} \times \cdots \times \mathscr{H}_{m}$. For every $i \in\{1, \cdots, m\}$ set, for every $n \in \mathbb{N}$,

$$
\begin{aligned}
x_{i, n+1}= & x_{i, n}+\lambda_{n}\left(\operatorname { p r o x } _ { \gamma _ { n } f _ { i } } \left(x_{i, n}-\gamma_{n}\left(\sum_{k=1}^{p} L_{k i}^{*} \nabla \varphi_{k}\left(\sum_{j=1}^{m} L_{k j} x_{j, n}\right)\right.\right.\right. \\
& \left.\left.\left.+b_{i, n}\right)\right)+a_{i, n}-x_{i, n}\right),
\end{aligned}
$$

where $\left(a_{i, n}\right)_{n \in \mathbb{N}}$ and $\left(b_{i, n}\right)_{n \in \mathbb{N}}$ are sequences in $\mathscr{H}_{i}$ such that

$$
\sum_{n \in \mathbb{N}}\left\|a_{i, n}\right\|<+\infty \text { and } \sum_{n \in \mathbb{N}}\left\|b_{i, n}\right\|<+\infty .
$$

Algorithm 1.1 generates $m$ sequences $\left(x_{1, n}\right)_{n \in \mathbb{N}}, \cdots,\left(x_{m, n}\right)_{n \in \mathbb{N}}$ in parallel. It also tolerates errors $a_{i, n}$ and $b_{i, n}$ in the implementation of the proximity operator and of the gradients, respectively. Its convergence to a solution to Problem 1.1 is guaranteed by the following theorem. Let us stress that, although some algorithms are available for specific instances of Problem 1.1 with $m=2$ (see [1,3,10], and [23, Section 4.4]), no method with such convergence properties seems to be available in the literature in the general setting we consider here.

Theorem 1.2. [4, Theorem 4.3] Let $\left(x_{1, n}\right)_{n \in \mathbb{N}}, \cdots,\left(x_{m, n}\right)_{n \in \mathbb{N}}$ be sequences generated by Algorithm 1.1. Then, for every $i \in\{1, \cdots, m\},\left(x_{i, n}\right)_{n \in \mathbb{N}}$ converges weakly to a point $x_{i} \in \mathscr{H}_{i}$, and $\left(x_{i}\right)_{1 \leq i \leq m}$ is a solution to Problem 1.1.

The paper is organized as follows. In Section 2, we introduce our notation and recall some important definitions and properties from convex analysis, and discuss proximity operators. In Section 3, we study the particular case when the coupling functions are Moreau envelopes and address specific situations. Section 4 is devoted to problems in which the coupling functions are quadratic. In Section 5, the focus is placed on quadratic coupling terms involving linear combinations of the components. Finally, Section 6 is devoted to an application to multiframe signal representation.

\section{Notation and background}

Throughout the paper, $\mathscr{H}$ and $\left(\mathscr{H}_{i}\right)_{1 \leq i \leq m}$ are real Hilbert spaces. Their scalar products are denoted by $\langle\cdot \mid \cdot\rangle$ and the associated norms by $\|\cdot\|$. Moreover, Id denotes the identity operator and $B(x ; \rho)$ the closed ball of center $x \in \mathscr{H}$ and radius $\rho \in] 0,+\infty[$. In this section, we recall some useful definitions and facts from convex analysis $[31,36,46]$ and provide background and new results on proximity operators.

\subsection{Convex analysis}

We denote by $\Gamma_{0}(\mathscr{H})$ the class of lower semicontinuous convex functions $\varphi: \mathscr{H} \rightarrow$ ]$-\infty,+\infty]$ which are proper in the sense that $\operatorname{dom} \varphi=\{x \in \mathscr{H} \mid \varphi(x)<+\infty\} \neq \varnothing$. 
Let $\varphi \in \Gamma_{0}(\mathscr{H})$. The set of minimizers of $\varphi$ is denoted by $\operatorname{Argmin} \varphi$ and, if $\varphi$ has a unique minimizer, this minimizer is denoted by $\operatorname{argmin}_{x \in \mathscr{H}} \varphi(x)$. The conjugate of $\varphi$ is the function $\varphi^{*} \in \Gamma_{0}(\mathscr{H})$ defined by

$$
\left.\left.\varphi^{*}: \mathscr{H} \rightarrow\right]-\infty,+\infty\right]: u \mapsto \sup _{x \in \mathscr{H}}\langle x \mid u\rangle-\varphi(x)
$$

and the subdifferential of $\varphi$ is the set-valued operator

$$
\partial \varphi: \mathscr{H} \rightarrow 2^{\mathscr{H}}: x \mapsto\{u \in \mathscr{H} \mid(\forall y \in \mathscr{H})\langle y-x \mid u\rangle+\varphi(x) \leq \varphi(y)\} .
$$

The Fenchel-Moreau theorem states that

$$
\varphi^{* *}=\varphi
$$

In addition,

$$
(\forall x \in \mathscr{H})(\forall u \in \mathscr{H}) \quad\left\{\begin{array}{l}
\varphi(x)+\varphi^{*}(u) \geq\langle x \mid u\rangle, \\
\varphi(x)+\varphi^{*}(u)=\langle x \mid u\rangle \Leftrightarrow u \in \partial \varphi(x) .
\end{array}\right.
$$

The next lemma follows directly from [17, Corollary 3.5].

Lemma 2.1. Let $g: \mathscr{H} \rightarrow\left[0,+\infty\left[\right.\right.$ be a continuous convex function and let $\phi \in \Gamma_{0}(\mathbb{R})$. Suppose that $\phi$ is increasing on $[0,+\infty[$ and that there exists a point $z \in \mathscr{H}$ such that $g(z) \in \operatorname{int} \operatorname{dom} \phi$. Then, for every $x \in \mathscr{H}$,

$$
\partial(\phi \circ g)(x)=\bigcup_{v \in \partial \phi(g(x)) \cap[0,+\infty[} v \partial g(x) .
$$

Now, let $C$ be a nonempty closed convex subset of $\mathscr{H}$. The indicator function of $C$ is

$$
\iota_{C}: x \mapsto \begin{cases}0, & \text { if } x \in C \\ +\infty, & \text { if } x \notin C\end{cases}
$$

the normal cone operator of $C$ is

$$
N_{C}=\partial \iota_{C}: x \mapsto \begin{cases}\{u \in \mathscr{H} \mid(\forall y \in C)\langle y-x \mid u\rangle \leq 0\}, & \text { if } x \in C, \\ \varnothing, & \text { otherwise; }\end{cases}
$$

the support function of $C$ is

$$
\left.\left.\sigma_{C}=\iota_{C}^{*}: \mathscr{H} \rightarrow\right]-\infty,+\infty\right]: u \mapsto \sup _{x \in C}\langle x \mid u\rangle,
$$

and the distance from $x \in \mathscr{H}$ to $C$ is $d_{C}(x)=\inf _{y \in C}\|x-y\|$. For every $x \in \mathscr{H}$, there exists a unique point $P_{C} x \in C$ such that $d_{C}(x)=\left\|x-P_{C} x\right\| ; P_{C} x$ is called the projection of $x$ onto $C$ and it is characterized by

$$
(\forall p \in \mathscr{H}) \quad p=P_{C} x \quad \Leftrightarrow \quad x-p \in N_{C} p
$$


We have

$$
(\forall x \in \mathscr{H}) \quad \partial d_{C}(x)= \begin{cases}\left\{\frac{x-P_{C} x}{d_{C}(x)}\right\}, & \text { if } \quad x \in \mathscr{H} \backslash C, \\ N_{C} x \cap B(0 ; 1), & \text { if } \quad x \in C .\end{cases}
$$

Lemma 2.2. Let $C$ be a nonempty convex closed subset of $\mathscr{H}$, let $\phi: \mathbb{R} \rightarrow]-\infty,+\infty]$ be increasing on $\left[0,+\infty\left[\right.\right.$ and even, and set $\varphi=\phi \circ d_{C}$. Then $\varphi^{*}=\sigma_{C}+\phi^{*} \circ\|\cdot\|$.

Proof. Set, for every $\eta \in\left[0,+\infty\left[, D_{\eta}=\{z \in \mathscr{H} \mid\|z\|=\eta\}\right.\right.$. For every $x \in \mathscr{H}$, since $\inf _{y \in C}\|x-y\|=\left\|x-P_{C} x\right\|$ and since $\phi$ is increasing on $[0,+\infty[$, we have

$$
(\forall z \in C) \quad \inf _{y \in C} \phi(\|x-y\|) \leq \phi\left(\left\|x-P_{C} x\right\|\right)=\phi\left(\inf _{y \in C}\|x-y\|\right) \leq \phi(\|x-z\|),
$$

which implies that inf $y \in C=(\|x-y\|)=\phi\left(\inf _{y \in C}\|x-y\|\right)$. Hence, since $\mathscr{H}=\bigcup_{\eta \in[0,+\infty[} D_{\eta}$ and since $\phi$ is even, we have

$$
\begin{aligned}
(\forall u \in \mathscr{H}) \quad \varphi^{*}(u) & =\sup _{x \in \mathscr{H}}\langle x \mid u\rangle-\phi\left(\inf _{y \in C}\|x-y\|\right) \\
& =\sup _{x \in \mathscr{H}}\langle x \mid u\rangle-\inf _{y \in C} \phi(\|x-y\|) \\
& =\sup _{y \in C} \sup _{x \in \mathscr{H}}\langle x \mid u\rangle-\phi(\|x-y\|) \\
& =\sup _{y \in C}\langle y \mid u\rangle+\sup _{z \in \mathscr{H}}\langle z \mid u\rangle-\phi(\|z\|) \\
& =\sup _{y \in C}\langle y \mid u\rangle+\sup _{\eta \in[0,+\infty[} \sup _{z \in D_{\eta}}\langle z \mid u\rangle-\phi(\eta) \\
& =\sup _{y \in C}\langle y \mid u\rangle+\sup _{\eta \in[0,+\infty[} \eta\|u\|-\phi(\eta) \\
& =\sup _{y \in C}\langle y \mid u\rangle+\sup _{\eta \in \mathbb{R}} \eta\|u\|-\phi(\eta) \\
& =\sigma_{C}(u)+\phi^{*}(\|u\|),
\end{aligned}
$$

which completes the proof.

Lemma 2.3. Let $\phi \in \Gamma_{0}(\mathbb{R})$ be such that $0 \in \operatorname{int} \operatorname{dom} \phi$, let $\left.\xi \in\right] 0,+\infty[\cap \operatorname{dom} \partial \phi$, and let $v \in \partial \phi(\xi)$. Then

$$
\max \partial \phi(0) \leq v .
$$

Proof. Since $0 \in \operatorname{int} \operatorname{dom} \phi, \partial \phi(0)$ is a nonempty compact set [36, p. 215 and Theorem 23.4]. Moreover, (2.2) yields

$$
(\forall \mu \in \partial \phi(0)) \quad\left\{\begin{array}{l}
(\xi-0) \mu+\phi(0) \leq \phi(\xi) \\
(0-\xi) v+\phi(\xi) \leq \phi(0)
\end{array}\right.
$$

Adding these inequalities results in

$$
(\forall \mu \in \partial \phi(0)) \quad \mu \xi \leq v \xi
$$

from which we deduce (2.13). 


\subsection{Proximity operators}

For a detailed account of the theory of proximity operators, see $[23,31]$ and the classical paper [35].

Let $\varphi \in \Gamma_{0}(\mathscr{H})$ and let $\left.\gamma \in\right] 0,+\infty[$. The Moreau envelope of index $\gamma$ of $\varphi$ is the continuous convex function

$$
\gamma_{\varphi}: \mathscr{H} \rightarrow \mathbb{R}: x \mapsto \inf _{y \in \mathscr{H}} \varphi(y)+\frac{1}{2 \gamma}\|x-y\|^{2} .
$$

For every $x \in \mathscr{H}$, the infimum in (2.16) is achieved at a unique point denoted by $\operatorname{prox}_{\gamma \varphi} x$, which is characterized by the inclusion

$$
(\forall p \in \mathscr{H}) \quad p=\operatorname{prox}_{\gamma \varphi} x \quad \Leftrightarrow \quad x-p \in \gamma \partial \varphi(p) .
$$

The proximity operator of $\varphi$ is defined as

$$
\operatorname{prox}_{\varphi}: \mathscr{H} \rightarrow \mathscr{H}: x \mapsto \operatorname{argmin}_{y \in \mathscr{H}} \varphi(y)+\frac{1}{2}\|x-y\|^{2} .
$$

The Moreau envelope ${ }^{\gamma} \varphi$ satisfies

$$
\gamma_{\varphi} \leq \varphi \text { and } \operatorname{Argmin}^{\gamma} \varphi=\operatorname{Argmin} \varphi .
$$

Moreover, it is Fréchet differentiable and

$$
\nabla^{\gamma} \varphi=\frac{1}{\gamma}\left(\operatorname{Id}-\operatorname{prox}_{\gamma \varphi}\right)=\operatorname{prox}_{\varphi^{*} / \gamma}(\cdot / \gamma) \text { is } 1 / \gamma \text {-Lipschitz continuous. }
$$

Lemma 2.4. [19, Proposition 11] Let $\mathscr{G}$ be a real Hilbert space, let $\psi \in \Gamma_{0}(\mathscr{G})$, let $L: \mathscr{H} \rightarrow$ $\mathscr{G}$ be linear and bounded, and set $\varphi=\psi \circ L$. Suppose that $L \circ L^{*}=\kappa \mathrm{Id}$, for some $\left.\kappa \in\right] 0,+\infty[$. Then $\varphi \in \Gamma_{0}(\mathscr{H})$ and

$$
\operatorname{prox}_{\varphi}=\operatorname{Id}+\frac{1}{\kappa} L^{*} \circ\left(\operatorname{prox}_{\kappa \psi}-\text { Id }\right) \circ L .
$$

If $C$ is a nonempty closed and convex subset $C$ of $\mathscr{H}$, we have

$$
\operatorname{prox}_{\gamma_{\iota_{C}}}=P_{C} .
$$

Closed-form expressions for the proximity operators of various functions can be found in $[16,19-21,23,35]$. We now derive new examples, some of which will be used in Section 3.3.

Proposition 2.1. Let $C$ be a nonempty closed convex subset of $\mathscr{H}$, let $\phi \in \Gamma_{0}(\mathbb{R})$ be even, and set $\varphi=\phi \circ d_{C}$. Then $\varphi \in \Gamma_{0}(\mathscr{H})$. Moreover, $\operatorname{prox}_{\varphi}=P_{C}$ if $\phi=\iota_{\{0\}}+\eta$ for some $\eta \in \mathbb{R}$ and, otherwise, for every $x \in \mathscr{H}$,

$$
\operatorname{prox}_{\varphi} x= \begin{cases}x+\frac{\operatorname{prox}_{\phi^{*}} d_{C}(x)}{d_{C}(x)}\left(P_{C} x-x\right), & \text { if } d_{C}(x)>\max \partial \phi(0), \\ P_{C} x, & \text { if } x \notin C \text { and } d_{C}(x) \leq \max \partial \phi(0), \\ x, & \text { if } x \in C .\end{cases}
$$


Proof. If $\phi=\iota_{\{0\}}+\eta$ for some $\eta \in \mathbb{R}$, then $\varphi=\iota_{C}+\eta$, which implies that $\varphi \in \Gamma_{0}(\mathscr{H})$ and that $\operatorname{prox}_{\varphi}=P_{C}$. Now assume that $\phi \neq \iota_{\{0\}}+\eta$ with $\eta \in \mathbb{R}$. Since $\phi$ is even, convex, and proper, we have $0 \in \operatorname{int} \operatorname{dom} \phi$ and it follows that

$$
(\forall z \in C) \quad d_{C}(z)=0 \in \operatorname{int} \operatorname{dom} \phi .
$$

Thus, $\varnothing \neq C \subset \operatorname{dom} \varphi$, which shows that $\varphi$ is proper. Next, since $d_{C}$ is continuous and $\phi$ is lower semicontinuous, $\varphi$ is lower semicontinuous. Moreover, since $\phi$ is convex and even, it is increasing on $\left[0,+\infty\right.$ [ and, by convexity of $d_{C}$, we deduce that $\varphi$ is convex. Altogether $\varphi \in \Gamma_{0}(\mathscr{H})$.

Now, let $x \in \mathscr{H}$ and set $p=\operatorname{prox}_{\varphi} x$. We derive from (2.17) that

$$
x-p \in \partial\left(\phi \circ d_{C}\right)(p) .
$$

Therefore, in view of (2.24), taking $g=d_{C}$ in Lemma 2.1 yields

$$
x-p \in \bigcup_{v \in \partial \phi\left(d_{C}(p)\right) \cap[0,+\infty[} v \partial d_{C}(p) .
$$

We examine two alternatives.

(a) $p \in C$ : In this case, $d_{C}(p)=0$ and, from (2.10), $\partial d_{C}(p)=N_{C} p \cap B(0 ; 1)$. Hence, (2.26) asserts that there exists $v \in \partial \phi(0) \cap[0,+\infty[$ such that

$$
x-p \in N_{C} p \cap B(0 ; v) .
$$

Using (2.9), we first deduce that

$$
p=P_{C} x
$$

In addition,

$$
d_{C}(x)=\left\|x-P_{C} x\right\|=\|x-p\| \leq v \leq \max \partial \phi(0) .
$$

(b) $p \notin C$ : In this case, $d_{C}(p)>0$ and (2.10) yields $\partial d_{C}(p)=\left\{\left(p-P_{C} p\right) / d_{C}(p)\right\}$. Hence, (2.26) implies that there exists $v \in \partial \phi\left(d_{C}(p)\right) \cap[0,+\infty$ [ such that

$$
x-p=v \frac{p-P_{C} p}{d_{C}(p)},
$$

which can be written equivalently as

$$
x-P_{C} p=\frac{v+d_{C}(p)}{d_{C}(p)}\left(p-P_{C} p\right) .
$$

Since (2.9) asserts that $p-P_{C} p \in N_{C}\left(P_{C} p\right)$, (2.31) yields $x-P_{C} p \in N_{C}\left(P_{C} p\right)$ and, therefore,

$$
P_{C} x=P_{C} p .
$$

Consequently, (2.31) is equivalent to

$$
x-P_{C} x=\frac{v+d_{C}(p)}{d_{C}(p)}\left(p-P_{C} p\right) .
$$


In turn, upon applying the norm, we obtain

$$
d_{C}(x)=v+d_{C}(p)
$$

Since $v \in \partial \phi\left(d_{C}(p)\right)$, we deduce from (2.34) that

$$
d_{C}(x)-d_{C}(p) \in \partial \phi\left(d_{C}(p)\right),
$$

which yields $d_{C}(p)=\operatorname{prox}_{\phi} d_{C}(x)$ by (2.17). Thus, it follows from (2.34) and (2.20) that

$$
v=\operatorname{prox}_{\phi^{*}}\left(d_{C}(x)\right) .
$$

On the other hand, it follows from (2.33) and (2.34) that

$$
\frac{p-P_{C} p}{d_{C}(p)}=\frac{x-P_{C} x}{v+d_{C}(p)}=\frac{x-P_{C} x}{d_{C}(x)} .
$$

Hence, using (2.36) and (2.37), we deduce from (2.30) that

$$
p=x+\frac{\operatorname{prox}_{\phi^{*}}\left(d_{C}(x)\right)}{d_{C}(x)}\left(P_{C} x-x\right) .
$$

In view of (2.28) and (2.38), it remains to show that

$$
p \in C \quad \Leftrightarrow \quad d_{C}(x) \leq \max \partial \phi(0) .
$$

To this end, we first observe that (2.29) yields $p \in C \Rightarrow d_{C}(x) \leq \max \partial \phi(0)$. For the reverse implication, suppose that $d_{C}(x) \leq \max \partial \phi(0)$ and that $p \notin C$. Then, we deduce from Lemma 2.3 and (2.34) that

$$
\max \partial \phi(0)+d_{C}(p) \leq v+d_{C}(p)=d_{C}(x) \leq \max \partial \phi(0),
$$

which implies that $d_{C}(p)=0$ and therefore that $p \in \bar{C}=C$, which contradicts our assumption.

Proposition 2.2. Let $C$ be a nonempty closed convex subset of $\mathscr{H}$, let $\phi \in \Gamma_{0}(\mathbb{R})$ be even and nonconstant, and set $\varphi=\sigma_{C}+\phi \circ\|\cdot\|$. Then $\varphi \in \Gamma_{0}(\mathscr{H})$ and, for every $x \in \mathscr{H}$,

$$
\operatorname{prox}_{\varphi} x= \begin{cases}\frac{\operatorname{prox}_{\phi} d_{C}(x)}{d_{C}(x)}\left(x-P_{C} x\right), & \text { if } d_{C}(x)>\max \operatorname{Argmin} \phi, \\ x-P_{C} x, & \text { if } x \notin C \text { and } d_{C}(x) \leq \max \operatorname{Argmin} \phi, \\ 0, & \text { if } x \in C .\end{cases}
$$

Proof. Set $\psi=\phi^{*} \circ d_{C}$. Since $\phi$ is an even function in $\Gamma_{0}(\mathbb{R}), \phi^{*}$ is likewise. Hence, it follows from Proposition 2.1 that $\psi \in \Gamma_{0}(\mathscr{H})$. Using the facts that $\partial \phi^{*}(0)=\operatorname{Argmin} \phi$, 
that $\phi^{*}$ is not of the form $\iota_{\{0\}}+\eta$ with $\eta \in \mathbb{R}$, and that, by (2.3), $\phi^{* *}=\phi$, we also derive from Proposition 2.1 that, for every $x \in \mathscr{H}$,

$$
\operatorname{prox}_{\psi} x= \begin{cases}x+\frac{\operatorname{prox}_{\phi} d_{C}(x)}{d_{C}(x)}\left(P_{C} x-x\right), & \text { if } d_{C}(x)>\max \operatorname{Argmin} \phi, \\ P_{C} x, & \text { if } x \notin C \text { and } d_{C}(x) \leq \max \operatorname{Argmin} \phi, \\ x, & \text { if } x \in C .\end{cases}
$$

On the other hand, Lemma 2.2 yields $\psi^{*}=\sigma_{C}+\phi^{* *} \circ\|\cdot\|=\sigma_{C}+\phi \circ\|\cdot\|=\varphi$. Hence, it follows from (2.20) (with $\gamma=1$ ) that $\operatorname{prox}_{\varphi}=\operatorname{prox}_{\psi^{*}}=\operatorname{Id}-\operatorname{prox}_{\psi}$. In view of (2.42), we thus obtain (2.41).

Proposition 2.3. Let $C$ be a nonempty closed convex subset of a real Hilbert space $\mathscr{G}$ and let $z \in \mathscr{G}$. Let $\phi \in \Gamma_{0}(\mathbb{R})$ be even and not of the form $\phi=\iota_{\{0\}}+\eta$ with $\eta \in \mathbb{R}$, let $L: \mathscr{H} \rightarrow \mathscr{G}$ be a bounded linear operator such that $L \circ L^{*}=\kappa \mathrm{Id}$ for some $\left.\kappa \in\right] 0,+\infty[$, and set

$$
\varphi: \mathscr{H} \rightarrow]-\infty,+\infty]: x \mapsto \phi\left(d_{C}(L x-z)\right) .
$$

Then $\varphi \in \Gamma_{0}(\mathscr{H})$ and, for every $x \in \mathscr{H}$,

$$
\operatorname{prox}_{\varphi} x=\left\{\begin{array}{c}
x+\frac{\operatorname{prox}_{(\kappa \phi)^{*}} d_{C}(L x-z)}{\kappa d_{C}(L x-z)} L^{*}\left(P_{C}(L x-z)+z-L x\right), \\
\text { if } d_{C}(L x-z)>\kappa \max \partial \phi(0), \\
x+\frac{1}{\kappa} L^{*}\left(P_{C}(L x-z)+z-L x\right), \\
x, \quad \text { if } L x-z \notin C \text { and } d_{C}(L x-z) \leq \kappa \max \partial \phi(0),
\end{array}\right.
$$

Proof. Set $g=\phi \circ d_{C}$. It follows from Proposition 2.1 that $g \in \Gamma_{0}(\mathscr{G})$ and that, for every $y \in \mathscr{G}$,

$$
\operatorname{prox}_{\kappa g} y= \begin{cases}y+\frac{\operatorname{prox}_{(\kappa \phi)^{*}} d_{C}(y)}{d_{C}(y)}\left(P_{C} y-y\right), & \text { if } d_{C}(y)>\kappa \max \partial \phi(0), \\ P_{C} y, & \text { if } d_{C}(y) \leq \kappa \max \partial \phi(0) .\end{cases}
$$

We also observe that, since $\varphi=g \circ(L \cdot-z)$ and $L$ is linear and continuous, $\varphi \in \Gamma_{0}(\mathscr{H})$. Now take $x \in \mathscr{H}$ and set $p=\operatorname{prox}_{\varphi} x$. Using (2.17), the identity $L \circ L^{*}=\kappa$ Id, and elementary subdifferential calculus, we obtain

$$
\begin{aligned}
p=\operatorname{prox}_{\varphi} x & \Leftrightarrow x-p \in \partial \varphi(p)=L^{*} \partial g(L p-z) \\
& \Leftrightarrow\left(x-\kappa^{-1} L^{*} z\right)-\left(p-\kappa^{-1} L^{*} z\right) \in L^{*} \partial g\left(L\left(p-\kappa^{-1} L^{*} z\right)\right) \\
& \Leftrightarrow\left(x-\kappa^{-1} L^{*} z\right)-\left(p-\kappa^{-1} L^{*} z\right) \in \partial(g \circ L)\left(p-\kappa^{-1} L^{*} z\right) \\
& \Leftrightarrow p-\kappa^{-1} L^{*} z=\operatorname{prox}_{g \circ L}\left(x-\kappa^{-1} L^{*} z\right) .
\end{aligned}
$$


Hence, by Lemma 2.4,

$$
\begin{aligned}
p & =\kappa^{-1} L^{*} z+\left(x-\kappa^{-1} L^{*} z\right)+\kappa^{-1} L^{*}\left(\operatorname{prox}_{\kappa g}\left(L\left(x-\kappa^{-1} L^{*} z\right)\right)-L\left(x-\kappa^{-1} L^{*} z\right)\right) \\
& =x+\kappa^{-1} L^{*}\left(\operatorname{prox}_{\kappa g}(L x-z)+z-L x\right) .
\end{aligned}
$$

Upon combining (2.47) and (2.45) we obtain (2.44).

\section{Coupling with Moreau envelopes}

In this section we interpret Problem 1.1 as a relaxation of a problem with a non-smooth coupling term.

\subsection{Problem formulation}

As seen in (2.20), the Moreau envelope of index $\left.\rho_{k} \in\right] 0,+\infty$ [ of a function $g_{k} \in \Gamma_{0}(\mathscr{G})$ is a convex function which is $1 / \rho_{k}$-Lipschitz differentiable everywhere. We can therefore set

$$
(\forall k \in\{1, \cdots, p\}) \quad \varphi_{k}={ }^{\rho_{k}} g_{k}
$$

in Problem 1.1 to obtain the following formulation.

Problem 3.1. Let $\left(\mathscr{H}_{i}\right)_{1 \leq i \leq m}$ and $\left(\mathscr{G}_{k}\right)_{1 \leq k \leq p}$ be real Hilbert spaces, and let $\left(\rho_{k}\right)_{1 \leq k \leq p}$ be in ] $0,+\infty\left[\right.$. For every $i \in\{1, \cdots, m\}$ and $k \in\{1, \cdots, p\}$, let $f_{i} \in \Gamma_{0}\left(\mathscr{H}_{i}\right)$, let $g_{k} \in \Gamma_{0}\left(\mathscr{G}_{k}\right)$, and let $L_{k i}: \mathscr{H}_{i} \rightarrow \mathscr{G}_{k}$ be linear and bounded. It is assumed that

$$
\min _{1 \leq k \leq p} \sum_{i=1}^{m}\left\|L_{k i}\right\|^{2}>0
$$

The problem is to

$$
\underset{x_{1} \in \mathscr{H}_{1}, \cdots, x_{m} \in \mathscr{H}_{m}}{\operatorname{minimize}} \sum_{i=1}^{m} f_{i}\left(x_{i}\right)+\sum_{k=1}^{p} \rho_{k} g_{k}\left(\sum_{i=1}^{m} L_{k i} x_{i}\right),
$$

under the assumption that solutions exist.

The functions $\left(\rho_{k} g_{k}\right)_{1 \leq k \leq p}$ are approximations to the functions $\left(g_{k}\right)_{1 \leq k \leq p}$ in the sense of (2.19). Thus, (3.3) can be regarded as a relaxation of the problem

$$
\underset{x_{1} \in \mathscr{H}_{1}, \cdots, x_{m} \in \mathscr{H}_{m}}{\operatorname{minimize}} \sum_{i=1}^{m} f_{i}\left(x_{i}\right)+\sum_{k=1}^{p} g_{k}\left(\sum_{i=1}^{m} L_{k i} x_{i}\right) .
$$

Since this problem involves not necessarily smooth coupling functions $\left(g_{k}\right)_{1 \leq k \leq p}$, it will in general be harder to solve than (3.3) and, in some cases, it may not possess any solution while (3.3) does (see [18] for an illustration of the latter situation).

In view of (3.1) and (2.20), the specialization of Algorithm 1.1 to Problem 3.1 assumes the following form. 
Algorithm 3.1. Set

$$
\beta_{1}=\frac{1}{p} \min _{1 \leq k \leq p} \frac{\rho_{k}}{\sum_{i=1}^{m}\left\|L_{k i}\right\|^{2}},
$$

and fix $\varepsilon$ in $] 0, \min \left\{1, \beta_{1}\right\}\left[,\left(\lambda_{n}\right)_{n \in \mathbb{N}}\right.$ in $[\varepsilon, 1],\left(\gamma_{n}\right)_{n \in \mathbb{N}}$ in $\left[\varepsilon, 2 \beta_{1}-\varepsilon\right]$, and $\left(x_{i, 0}\right)_{1 \leq i \leq m}$ in $\mathscr{H}_{1} \times \cdots \times \mathscr{H}_{m}$. For every $i \in\{1, \cdots, m\}$ set, for every $n \in \mathbb{N}$,

$$
\begin{aligned}
x_{i, n+1}= & x_{i, n}+\lambda_{n}\left(\operatorname { p r o x } _ { \gamma _ { n } f _ { i } } \left(x_{i, n}+\gamma_{n}\left(\sum_{k=1}^{p} L_{k i}^{*}\left(\frac{\operatorname{prox}_{\rho_{k} g_{k}}-\mathrm{Id}}{\rho_{k}}\right)\left(\sum_{j=1}^{m} L_{k j} x_{j, n}\right)\right.\right.\right. \\
& \left.\left.\left.+b_{i, n}\right)\right)+a_{i, n}-x_{i, n}\right),
\end{aligned}
$$

where $\left(a_{i, n}\right)_{n \in \mathbb{N}}$ and $\left(b_{i, n}\right)_{n \in \mathbb{N}}$ are sequences in $\mathscr{H}_{i}$ such that

$$
\sum_{n \in \mathbb{N}}\left\|a_{i, n}\right\|<+\infty, \quad \sum_{n \in \mathbb{N}}\left\|b_{i, n}\right\|<+\infty
$$

We obtain the weak convergence of this algorithm as a direct application of Theorem 1.2 .

Corollary 3.1. Let $\left(x_{1, n}\right)_{n \in \mathbb{N}}, \cdots,\left(x_{m, n}\right)_{n \in \mathbb{N}}$ be sequences generated by Algorithm 3.1. Then, for every $i \in\{1, \cdots, m\},\left(x_{i, n}\right)_{n \in \mathbb{N}}$ converges weakly to a point $x_{i} \in \mathscr{H}_{i}$, and $\left(x_{i}\right)_{1 \leq i \leq m}$ is a solution to Problem 3.1.

Remark 3.1. In the particular case of $m=1$ variable, Problem 3.1 reduces to [23, Problem 4.1], which was itself shown in [23, Section 4] to cover several signal decomposition and recovery problems.

\subsection{Relaxation of problems with hard coupling}

As a first application of the results of Section 3.1, we consider problems in which hard constraints on $p$ linear mixtures of the signals are available. More precisely, the constraints are of the form

$$
(\forall k \in\{1, \cdots, p\}) \quad \sum_{i=1}^{m} L_{k i} x_{i} \in D_{k}
$$

where each $D_{k}$ is a nonempty closed convex subset of $\mathscr{G}_{k}$ and, for every $i \in\{1, \cdots, m\}, L_{k i}$ is a bounded linear operator from $\mathscr{H}_{i}$ to $\mathscr{G}_{k}$. In our setting, this leads to the hard-coupled problem

$$
\underset{\substack{x_{1} \in \mathscr{H}_{1}, \cdots, x_{m} \in \mathscr{H}_{m} \\ \sum_{i=1}^{m} L_{1 i} x_{i} \in D_{1}, \cdots, \sum_{i=1}^{m} L_{p i} x_{i} \in D_{p}}}{\operatorname{minimize}} \sum_{i=1}^{m} f_{i}\left(x_{i}\right),
$$

which amounts to setting

$$
(\forall k \in\{1, \cdots, p\}) \quad g_{k}=\iota_{D_{k}}
$$


in (3.4). Let us note that, due to inaccuracies in the definition of the sets $\left(D_{k}\right)_{1 \leq k \leq p}$ $[18,29]$, (3.9) may be infeasible in the sense that $\bigcap_{k=1}^{p} D_{k}=\varnothing$. On the other hand, the approximate problem (3.3), which becomes

$$
\underset{x_{1} \in \mathscr{H}_{1}, \cdots, x_{m} \in \mathscr{H}_{m}}{\operatorname{minimize}} \sum_{i=1}^{m} f_{i}\left(x_{i}\right)+\sum_{k=1}^{p} \frac{1}{2 \rho_{k}} d_{D_{k}}^{2}\left(\sum_{i=1}^{m} L_{k i} x_{i}\right),
$$

will admit solutions under mild assumptions [23, Proposition 3.1(i)]. Moreover, using (2.22), the iteration (3.6) in Algorithm 3.1 reduces to (we set $a_{i, n} \equiv 0, b_{i, n} \equiv 0$, and $\lambda_{n} \equiv 1$ for simplicity)

$$
x_{i, n+1}=\operatorname{prox}_{\gamma_{n} f_{i}}\left(x_{i, n}+\gamma_{n} \sum_{k=1}^{p} L_{k i}^{*}\left(\frac{P_{D_{k}}-\mathrm{Id}}{\rho_{k}}\right)\left(\sum_{j=1}^{m} L_{k j} x_{j, n}\right)\right) .
$$

As an illustration of the construction of the sets $\left(D_{k}\right)_{1 \leq k \leq p}$, let us consider the problem of finding $m$ sources $\left(x_{i}\right)_{1 \leq i \leq m}$ from the noisy observation of $p$ mixtures

$$
(\forall k \in\{1, \cdots, p\}) \quad z_{k}=\sum_{i=1}^{m} L_{k i} x_{i}+w_{k},
$$

where $w_{k} \in \mathscr{G}_{k}$ represents the noise corrupting the $k$ th measurement. As discussed in $[22,39]$, a wide range of probabilistic a priori information on the $k$ th noise process can be translated into constraints of the form $z_{k}-\sum_{i=1}^{m} L_{k i} x_{i} \in E_{k}$, where $E_{k}$ is a closed convex subset of $\mathscr{G}_{k}$. This corresponds to (3.8), where $D_{k}=z_{k}-E_{k}$. For instance, if a statistical bound $\eta_{k}$ is available on the energy of the $k$ th noise process, we obtain $D_{k}=B\left(z_{k} ; \sqrt{\eta_{k}}\right)$.

\subsection{Relaxation of problems with hard constraints and hard coupling}

We place ourselves in the same setting as in Section 3.2 and make the additional assumption that hard constraints are available for each signal, namely

$$
(\forall i \in\{1, \cdots, m\}) \quad x_{i} \in C_{i},
$$

where each $C_{i}$ is a nonempty closed convex subset of $\mathscr{H}_{i}$. In this context, (3.9) coincides with the feasibility problem

$$
\text { Find } x_{1} \in C_{1}, \cdots, x_{m} \in C_{m} \text { such that } \sum_{i=1}^{m} L_{1 i} x_{i} \in D_{1}, \cdots, \sum_{i=1}^{m} L_{p i} x_{i} \in D_{p} \text {. }
$$

Let us relax the $p$ constraints $\sum_{i=1}^{m} L_{k i} x_{i} \in D_{k}$ as in (3.11) and the $m$ constraints in (3.14) by penalizing the distances to the sets via functions

$$
(\forall i \in\{1, \cdots, m\}) \quad f_{i}=\phi_{i} \circ d_{C_{i}}
$$


where $\left(\phi_{i}\right)_{1 \leq i \leq m}$ are nonzero even functions in $\Gamma_{0}(\mathbb{R}) \backslash\left\{\iota_{\{0\}}\right\}$ such that $\phi_{i}(0) \equiv 0$. Thus, (3.11) becomes

$$
\underset{x_{1} \in \mathscr{H}_{1}, \cdots, x_{m} \in \mathscr{H}_{m}}{\operatorname{minimize}} \sum_{i=1}^{m} \phi_{i}\left(d_{C_{i}}\left(x_{i}\right)\right)+\sum_{k=1}^{p} \frac{1}{2 \rho_{k}} d_{D_{k}}^{2}\left(\sum_{i=1}^{m} L_{k i} x_{i}\right),
$$

which is our relaxation of (3.15). Corollary 3.1 asserts that this problem can be solved via Algorithm 3.1 where, by virtue of Proposition 2.1, (3.6) reduces to (we set $a_{i, n} \equiv 0$, $b_{i, n} \equiv 0$, and $\lambda_{n} \equiv 1$ for simplicity)

$$
\left\{\begin{array}{l}
y_{i, n}=x_{i, n}+\gamma_{n} \sum_{k=1}^{p} L_{k i}^{*}\left(\frac{P_{D_{k}}-\text { Id }}{\rho_{k}}\right)\left(\sum_{j=1}^{m} L_{k j} x_{j, n}\right) \\
x_{i, n+1}= \begin{cases}y_{i, n}+\frac{\operatorname{prox}_{\left(\gamma_{n} \phi_{i}\right)^{*}} d_{C_{i}}\left(y_{i, n}\right)}{d_{C_{i}}\left(y_{i, n}\right)}\left(P_{C_{i}} y_{i, n}-y_{i, n}\right), \\
\text { if } d_{C_{i}}\left(y_{i, n}\right)>\gamma_{n} \max \partial \phi_{i}(0), \\
P_{C_{i}} y_{i, n}, & \text { if } y_{i, n} \notin C_{i} \text { and } d_{C_{i}}\left(y_{i, n}\right) \leq \gamma_{n} \max \partial \phi_{i}(0), \\
y_{i, n}, & \text { if } y_{i, n} \in C_{i} .\end{cases}
\end{array}\right.
$$

\section{Quadratic coupling} form

In this section, we study Problem 1.1 when the coupling functions $\left(\varphi_{k}\right)_{1 \leq k \leq p}$ are of the

$$
(\forall k \in\{1, \cdots, p\}) \quad \varphi_{k}=\frac{1}{2}\left\|z_{k}-\cdot\right\|^{2}, \quad \text { where } \quad z_{k} \in \mathscr{G}_{k} .
$$

\subsection{Problem formulation}

We first restate Problem 1.1 under assumption (4.1).

Problem 4.1. Let $\left(\mathscr{H}_{i}\right)_{1 \leq i \leq m}$ and $\left(\mathscr{G}_{k}\right)_{1 \leq k \leq p}$ be real Hilbert spaces. For every $i \in\{1, \cdots, m\}$, let $f_{i} \in \Gamma_{0}\left(\mathscr{H}_{i}\right)$ and, for every $k \in\{1, \cdots, p\}$, let $z_{k} \in \mathscr{G}_{k}$ and let $L_{k i}: \mathscr{H}_{i} \rightarrow \mathscr{G}_{k}$ be linear and bounded. It is assumed that $\min _{1 \leq k \leq p} \sum_{i=1}^{m}\left\|L_{k i}\right\|^{2}>0$. The problem is to

$$
\underset{x_{1} \in \mathscr{H}_{1}, \cdots, x_{m} \in \mathscr{H}_{m}}{\operatorname{minimize}} \sum_{i=1}^{m} f_{i}\left(x_{i}\right)+\frac{1}{2} \sum_{k=1}^{p}\left\|z_{k}-\sum_{i=1}^{m} L_{k i} x_{i}\right\|^{2},
$$

under the assumption that solutions exist.

Here is a variant of Algorithm 1.1 for solving Problem 4.1.

Algorithm 4.1. Set

$$
\beta_{2}=\frac{1}{\sum_{k=1}^{p} \sum_{i=1}^{m}\left\|L_{k i}\right\|^{2}}
$$


and fix $\varepsilon$ in $] 0, \min \left\{1, \beta_{2}\right\}\left[,\left(\lambda_{n}\right)_{n \in \mathbb{N}}\right.$ in $[\varepsilon, 1]\left(\gamma_{n}\right)_{n \in \mathbb{N}}$ in $\left[\varepsilon, 2 \beta_{2}-\varepsilon\right]$, and $\left(x_{i, 0}\right)_{1 \leq i \leq m}$ in $\mathscr{H}_{1} \times \cdots \times \mathscr{H}_{m}$. For every $i \in\{1, \cdots, m\}$ set, for every $n \in \mathbb{N}$,

$$
\begin{aligned}
x_{i, n+1}= & x_{i, n}+\lambda_{n}\left(\operatorname { p r o x } _ { \gamma _ { n } f _ { i } } \left(x_{i, n}+\gamma_{n}\left(\sum_{k=1}^{p} L_{k i}^{*}\left(z_{k}-\sum_{j=1}^{m} L_{k j} x_{j, n}\right)\right.\right.\right. \\
& \left.\left.\left.+b_{i, n}\right)\right)+a_{i, n}-x_{i, n}\right),
\end{aligned}
$$

where $\left(a_{i, n}\right)_{n \in \mathbb{N}}$ and $\left(b_{i, n}\right)_{n \in \mathbb{N}}$ are sequences in $\mathscr{H}_{i}$ such that

$$
\sum_{n \in \mathbb{N}}\left\|a_{i, n}\right\|<+\infty, \quad \sum_{n \in \mathbb{N}}\left\|b_{i, n}\right\|<+\infty .
$$

Remark 4.1. The Lipschitz constant of each $\nabla \varphi_{k}$ is $\tau_{k}=1$. Hence, the bound $\beta_{1}$ of (1.4) is $\beta_{1}=1 /\left(p \max _{1 \leq k \leq p} \sum_{i=1}^{m}\left\|L_{k i}\right\|^{2}\right)$. If we used this bound in (4.3), we could derive at once the convergence of Algorithm 4.1 from Theorem 1.2. However, we use the bound $\beta_{2}$ of (4.3), which is better than the general bound $\beta_{1}$ since

$$
\beta_{1}=\left(p \max _{1 \leq k \leq p} \sum_{i=1}^{m}\left\|L_{k i}\right\|^{2}\right)^{-1} \leq\left(\sum_{k=1}^{p} \sum_{i=1}^{m}\left\|L_{k i}\right\|^{2}\right)^{-1}=\beta_{2} .
$$

Theorem 4.2. Let $\left(x_{1, n}\right)_{n \in \mathbb{N}}, \cdots,\left(x_{m, n}\right)_{n \in \mathbb{N}}$ be sequences generated by Algorithm 4.1. Then, for every $i \in\{1, \cdots, m\},\left(x_{i, n}\right)_{n \in \mathbb{N}}$ converges weakly to a point $x_{i} \in \mathscr{H}_{i}$, and $\left(x_{i}\right)_{1 \leq i \leq m}$ is a solution to Problem 4.1.

Proof. We set $\mathscr{H}=\mathscr{H}_{1} \oplus \cdots \oplus \mathscr{H}_{m}$, i.e., $\mathscr{H}$ is the real Hilbert space obtained by endowing $\mathscr{H}_{1} \times \cdots \times \mathscr{H}_{m}$ with the scalar product $\langle\langle\cdot \mid \cdot\rangle\rangle:(\boldsymbol{x}, \boldsymbol{y}) \mapsto \sum_{i=1}^{m}\left\langle x_{i} \mid y_{i}\right\rangle$, with associated norm $\|\cdot \mid\|: x \mapsto \sqrt{\sum_{i=1}^{m}\left\|x_{i}\right\|^{2}}$, where $\boldsymbol{x}=\left(x_{i}\right)_{1 \leq i \leq m}$ denotes a generic element in $\mathscr{H}$. We also introduce

$$
\boldsymbol{g}: \mathscr{H} \rightarrow \mathbb{R}: \boldsymbol{x} \mapsto \frac{1}{2} \sum_{k=1}^{p}\left\|z_{k}-\sum_{j=1}^{m} L_{k j} x_{j}\right\|^{2},
$$

and, for every $i \in\{1, \cdots, m\}$, we let $B_{i}$ be the gradient of $g$ with respect to the $i$ th variable. Thus, $\nabla \boldsymbol{g}=\left(B_{i}\right)_{1 \leq i \leq m}$, where

$$
B_{i}: \mathscr{H} \rightarrow \mathscr{H}_{i}: \boldsymbol{x} \mapsto \sum_{k=1}^{p} L_{k i}^{*}\left(\sum_{j=1}^{m} L_{k j} x_{j}-z_{k}\right) .
$$

Now take $\boldsymbol{x}$ and $\boldsymbol{y}$ in $\mathscr{H}$. Since, in view of (4.1), (4.4) is a special case of (1.5), proceeding as in the proof of [4, Theorem 4.3], to reach the announced conclusion it is enough to show that

$$
\sum_{i=1}^{m}\left\langle B_{i}(\boldsymbol{x})-B_{i}(\mathbf{y}) \mid x_{i}-y_{i}\right\rangle \geq \beta_{2} \sum_{i=1}^{m}\left\|B_{i}(\boldsymbol{x})-B_{i}(\mathbf{y})\right\|^{2},
$$


or, equivalently, that $\langle\langle\nabla \boldsymbol{g}(\boldsymbol{x})-\nabla \boldsymbol{g}(\boldsymbol{y}) \mid \boldsymbol{x}-\boldsymbol{y}\rangle\rangle \geq \beta_{2}|\|\nabla \boldsymbol{g}(\boldsymbol{x})-\nabla \boldsymbol{g}(\boldsymbol{y})\||^{2}$. Since $\boldsymbol{g}$ is convex, it follows from the Baillon-Haddad theorem [9, Corollary 10] that this inequality is equivalent to $|\|\nabla \boldsymbol{g}(\boldsymbol{x})-\nabla \boldsymbol{g}(\boldsymbol{y})\|| \leq\|\boldsymbol{x}-\boldsymbol{y}\||| / \beta_{2}$, i.e., to

$$
\sum_{i=1}^{m}\left\|B_{i}(\boldsymbol{x})-B_{i}(\boldsymbol{y})\right\|^{2} \leq\|\| \boldsymbol{x}-\boldsymbol{y}\|\|^{2} / \beta_{2}^{2} .
$$

For every $i \in\{1, \cdots, m\},(4.8)$ and the Cauchy-Schwarz inequality imply that

$$
\begin{aligned}
\left\|B_{i}(\boldsymbol{x})-B_{i}(\boldsymbol{y})\right\|^{2} & =\left\|\sum_{j=1}^{m} \sum_{k=1}^{p} L_{k i}^{*} L_{k j}\left(x_{j}-y_{j}\right)\right\|^{2} \\
& \leq\left(\sum_{j=1}^{m} \sum_{k=1}^{p}\left\|L_{k i}\right\|\left\|L_{k j}\right\|\left\|x_{j}-y_{j}\right\|\right)^{2} \\
& =\left(\sum_{k=1}^{p}\left\|L_{k i}\right\| \sum_{j=1}^{m}\left\|L_{k j}\right\|\left\|x_{j}-y_{j}\right\|\right)^{2} \\
& \leq\left(\sum_{k=1}^{p}\left\|L_{k i}\right\|^{2}\right) \sum_{k=1}^{p}\left(\sum_{j=1}^{m}\left\|L_{k j}\right\|\left\|x_{j}-y_{j}\right\|\right)^{2} \\
& \leq\left(\sum_{k=1}^{p}\left\|L_{k i}\right\|^{2}\right) \sum_{k=1}^{p}\left(\sum_{j=1}^{m}\left\|L_{k j}\right\|^{2}\right)\left(\sum_{j=1}^{m}\left\|x_{j}-y_{j}\right\|^{2}\right) \\
& =\left.\left(\sum_{k=1}^{p}\left\|L_{k i}\right\|^{2}\right)\|\boldsymbol{x}-\boldsymbol{y}\|\right|^{2} / \beta_{2} .
\end{aligned}
$$

Hence,

$$
\sum_{i=1}^{m}\left\|B_{i}(\boldsymbol{x})-B_{i}(\boldsymbol{y})\right\|^{2} \leq\left(\sum_{i=1}^{m} \sum_{k=1}^{p}\left\|L_{k i}\right\|^{2}\right)\|\mid \boldsymbol{x}-\boldsymbol{y}\|^{2} / \beta_{2}=\|\boldsymbol{x}-\boldsymbol{y}\| \|^{2} / \beta_{2}^{2},
$$

which yields (4.10).

\subsection{Split feasibility problems}

Suppose that $m=p+1$. For every $k \in\{1, \cdots, p\}$, set $z_{k}=0, \mathscr{G}_{k}=\mathscr{H}_{k+1}$, and, for every $i \in\{2, \cdots, m\}$,

$$
L_{k i}= \begin{cases}-\mathrm{Id}, & \text { if } i=k+1, \\ 0, & \text { otherwise. }\end{cases}
$$

Then (4.2) becomes

$$
\underset{x_{1} \in \mathscr{H}_{1}, \cdots, x_{m} \in \mathscr{H}_{m}}{\operatorname{minimize}} \sum_{i=1}^{m} f_{i}\left(x_{i}\right)+\frac{1}{2} \sum_{k=1}^{m-1}\left\|L_{k 1} x_{1}-x_{k+1}\right\|^{2} .
$$


Setting errors to zero and $\lambda_{n} \equiv 1$ for simplicity, the updating rule (4.4) in Algorithm 4.1 reduces to

$$
\left\{\begin{array}{l}
x_{1, n+1}=\operatorname{prox}_{\gamma_{n} f_{1}}\left(x_{1, n}-\gamma_{n} \sum_{k=1}^{m-1} L_{k 1}^{*}\left(L_{k 1} x_{1, n}-x_{k+1, n}\right)\right) \\
x_{i, n+1}=\operatorname{prox}_{\gamma_{n} f_{i}}\left(\left(1-\gamma_{n}\right) x_{i, n}+\gamma_{n} L_{i-1,1} x_{1, n}\right), \quad \text { for } \quad 2 \leq i \leq m .
\end{array}\right.
$$

In particular, if each $f_{i}$ in (4.14) is the indicator function of a nonempty closed convex set $C_{i} \subset \mathscr{H}_{1}$, we obtain

$$
\underset{x_{1} \in C_{1}, \cdots, x_{m} \in C_{m}}{\operatorname{minimize}} \frac{1}{2} \sum_{k=1}^{m-1}\left\|L_{k 1} x_{1}-x_{k+1}\right\|^{2} \text {, }
$$

which can be regarded as a relaxation of the split feasibility problem

$$
\text { find } x_{1} \in C_{1} \text { such that } L_{11} x_{1} \in C_{2}, L_{21} x_{1} \in C_{3}, \cdots, L_{m-1,1} x_{1} \in C_{m} \text {. }
$$

For $m=2$, this type of problem was introduced in [13] and further studied in [11,14,23].

In [42] a problem similar to (4.14) is investigated in the case when $m=2$, in which the linear operator depends on the partial derivatives of one component.

\section{Strong coupling}

An important instance of Problem 4.1 occurs when the linear mixtures describing the interactions between the components in (4.2) reduce to linear combinations. Such a coupling is referred to as strong.

\subsection{Problem formulation}

Problem 5.1. For every $i \in\{1, \cdots, m\}$, let $f_{i} \in \Gamma_{0}(\mathscr{H})$ and, for every $k \in\{1, \cdots, p\}$, let $\xi_{k i} \in \mathbb{R}$ and let $z_{k} \in \mathscr{H}$. It is assumed that $\min _{1 \leq k \leq p} \sum_{i=1}^{m}\left|\xi_{k i}\right|>0$. The problem is to

$$
\underset{x_{1} \in \mathscr{H}, \cdots, x_{m} \in \mathscr{H}}{\operatorname{minimize}} \sum_{i=1}^{m} f_{i}\left(x_{i}\right)+\frac{1}{2} \sum_{k=1}^{p}\left\|z_{k}-\sum_{i=1}^{m} \xi_{k i} x_{i}\right\|^{2},
$$

under the assumption that solutions exist.

To solve this problem, we propose the following variant of Algorithm 4.1, which features a better bound than (4.3).

Algorithm 5.1. Set $\Xi=\left[\xi_{k i}\right] \in \mathbb{R}^{p \times m}, \Delta=\Xi^{\top} \Xi=\left[\delta_{i j}\right]$, and

$$
\beta_{3}=\frac{1}{\lambda_{\max }},
$$


where $\lambda_{\max }$ is the largest eigenvalue of $\Delta$. Fix $\varepsilon$ in $] 0, \min \left\{1, \beta_{3}\right\}\left[,\left(\lambda_{n}\right)_{n \in \mathbb{N}}\right.$ in $[\varepsilon, 1],\left(\gamma_{n}\right)_{n \in \mathbb{N}}$ in $\left[\varepsilon, 2 \beta_{3}-\varepsilon\right]$, and $\left(x_{i, 0}\right)_{1 \leq i \leq m}$ in $\mathscr{H}^{m}$. For every $i \in\{1, \cdots, m\}$ set, for every $n \in \mathbb{N}$,

$$
x_{i, n+1}=x_{i, n}+\lambda_{n}\left(\operatorname{prox}_{\gamma_{n} f_{i}}\left(x_{i, n}+\gamma_{n} \sum_{k=1}^{p} \xi_{k i}\left(z_{k}-\sum_{j=1}^{m} \xi_{k j} x_{j, n}\right)\right)+a_{i, n}-x_{i, n}\right) \text {, }
$$

where $\left(a_{i, n}\right)_{n \in \mathbb{N}}$ is a sequence in $\mathscr{H}$ such that $\sum_{n \in \mathbb{N}}\left\|a_{i, n}\right\|<+\infty$.

Remark 5.1. Using standard matrix norm inequalities, we obtain

$$
\lambda_{\max } \leq \sum_{k=1}^{p} \sum_{i=1}^{m}\left|\xi_{k i}\right|^{2} \leq p \max _{1 \leq k \leq p} \sum_{i=1}^{m}\left|\xi_{k i}\right|^{2}
$$

which yields

$$
\beta_{3}=\frac{1}{\lambda_{\max }} \geq \beta_{2}=\frac{1}{\sum_{k=1}^{p} \sum_{i=1}^{m}\left|\xi_{k i}\right|^{2}} \geq \beta_{1}=\frac{1}{p \max _{1 \leq k \leq p} \sum_{i=1}^{m}\left|\xi_{k i}\right|^{2}} .
$$

In other words, in the problem under consideration, the bound of (5.2) is better than that of (4.3), which is itself better than that of (1.4).

Theorem 5.2. Let $\left(x_{1, n}\right)_{n \in \mathbb{N}}, \cdots,\left(x_{m, n}\right)_{n \in \mathbb{N}}$ be sequences generated by Algorithm 5.1. Then, for every $i \in\{1, \cdots, m\},\left(x_{i, n}\right)_{n \in \mathbb{N}}$ converges weakly to a point $x_{i} \in \mathscr{H}$, and $\left(x_{i}\right)_{1 \leq i \leq m}$ is a solution to Problem 5.1.

Proof. Define $\mathscr{H}$ as in the proof of Theorem 4.2 (with $\mathscr{H}_{i} \equiv \mathscr{H}$ ), set

$$
\boldsymbol{g}: \mathscr{H} \rightarrow \mathbb{R}: \boldsymbol{x} \mapsto \frac{1}{2} \sum_{k=1}^{p}\left\|z_{k}-\sum_{i=1}^{m} \xi_{k i} x_{i}\right\|^{2},
$$

and introduce the bounded linear operator

$$
\text { B: } \mathscr{H} \rightarrow \mathscr{H}: \boldsymbol{x} \mapsto\left(\sum_{k=1}^{p} \sum_{j=1}^{m} \xi_{k i} \xi_{k j} x_{j}\right)_{1 \leq i \leq m} .
$$

As in the proof of Theorem 4.2, it is sufficient to prove that $\nabla g$ is $\beta_{3}^{-1}$-Lipschitz continuous.

Since $\Delta$ is a real $m \times m$ symmetric matrix, there exists an orthogonal matrix $\Pi=$ $\left[\pi_{i j}\right] \in \mathbb{R}^{m \times m}$ such that $\Delta=\Pi \Lambda \Pi^{\top}$, where $\Lambda$ is the diagonal matrix the diagonal entries of which are the eigenvalues $\left(\lambda_{i}\right)_{1 \leq i \leq m}$ of $\Delta$. Now set $\boldsymbol{D}: \mathscr{H} \rightarrow \mathscr{H}: \boldsymbol{x} \mapsto\left(\lambda_{i} x_{i}\right)_{1 \leq i \leq m}$ and $\boldsymbol{U}: \mathscr{H} \rightarrow \mathscr{H}: \boldsymbol{x} \mapsto\left(\sum_{j=1}^{m} \pi_{i j} x_{j}\right)_{1 \leq i \leq m}$. Then $\boldsymbol{U}$ is unitary and

$$
\left.\|\boldsymbol{B}\|\right|^{2}=\|\| \boldsymbol{D} \boldsymbol{U}^{*}\left\|\left.\right|^{2}=\right\| \boldsymbol{D}\left\|\left.\right|^{2}=\sup _{\|\boldsymbol{x}\| \leq 1} \sum_{i=1}^{m} \lambda_{i}^{2}\right\| x_{i} \|^{2}=\lambda_{\text {max }}^{2} .
$$

Hence, for every $\boldsymbol{x}$ and $\boldsymbol{y}$ in $\mathscr{H}$, we have

$$
\left.\left\|\left.|\nabla \boldsymbol{g}(\boldsymbol{x})-\nabla \boldsymbol{g}(\boldsymbol{y})|\right|^{2}=\right\|\|\boldsymbol{B} \boldsymbol{x}-\boldsymbol{B} \boldsymbol{y}\|\right|^{2} \leq \lambda_{\max }^{2}|\|\boldsymbol{x}-\boldsymbol{y}\||^{2},
$$

which implies that $\nabla \boldsymbol{g}$ is $\beta_{3}^{-1}$-Lipschitz continuous and completes the proof. 


\subsection{Signal decomposition}

Suppose that an ideal signal $\bar{x} \in \mathscr{H}$ can be decomposed as

$$
\bar{x}=\sum_{i=1}^{m} \bar{x}_{i}, \quad \text { where } \quad(\forall i \in\{1, \cdots, m\}) \quad \bar{x}_{i} \in \mathscr{H} .
$$

A common problem is to recover the components $\left(\bar{x}_{i}\right)_{1 \leq i \leq m}$ from some measurement $z$ of $\bar{x}$ and some prior information. Assuming that the prior information on each component $\bar{x}_{i}$ is promoted by a potential $f_{i} \in \Gamma_{0}(\mathscr{H})$ and using a least-squares data fitting term leads to the variational problem

$$
\underset{x_{1} \in \mathscr{H}, \cdots, x_{m} \in \mathscr{H}}{\operatorname{minimize}} \sum_{i=1}^{m} f_{i}\left(x_{i}\right)+\frac{1}{2}\left\|z-\sum_{i=1}^{m} x_{i}\right\|^{2} .
$$

Instances of this problem have been considered in $[5,7,23,41,42]$ for $m=2$, in $[6,27]$ for $m=3$, and in [15] for $m=4$.

We observe that (5.11) is a special case of (5.1), where $p=1, z_{1}=z$, and, for every $i \in\{1, \cdots, m\}, \xi_{1 i}=1$. Thus, $\Xi=[1 \cdots 1] \in \mathbb{R}^{1 \times m}$ and $\beta_{3}=1 / m$ in Algorithm 5.1. Moreover, the updating rule (5.3) now assumes the form

$$
x_{i, n+1}=x_{i, n}+\lambda_{n}\left(\operatorname{prox}_{\gamma_{n} f_{i}}\left(x_{i, n}+\gamma_{n}\left(z-\sum_{j=1}^{m} x_{j, n}\right)\right)+a_{i, n}-x_{i, n}\right) .
$$

The weak convergence of the $m$ sequences so generated to a solution to (5.11) is guaranteed by Theorem 5.2. For $m=2$, an alternative weakly convergent method is proposed in [23, Section 4.4], which subsumes that of [5] (see also the alternative method of [3]). However, for $m>2$, no weakly convergent algorithm seems to be available in the literature. Thus, in [15], a model of the form (5.11) with $m=4$ component is investigated but no convergence proof is furnished for the proposed cyclic minimization algorithm; in [6], a model with $m=3$ components is investigated in $\mathscr{H}=\mathbb{R}^{N}$ and a coordinate descent algorithm with modest convergence properties is utilized.

For the sake of illustration, consider the case when $m=3$. Then (5.2) yields $\beta=1 / 3$. Taking for simplicity $\gamma_{n} \equiv 1 / 2, \lambda_{n} \equiv 1$, and, for every $i \in\{1,2,3\}, a_{i, n} \equiv 0$, (5.12) leads to the simple parallel scheme

$$
\left\{\begin{array}{l}
x_{1, n+1}=\operatorname{prox}_{f_{1} / 2}\left(\left(z+x_{1, n}-x_{2, n}-x_{3, n}\right) / 2\right) \\
x_{2, n+1}=\operatorname{prox}_{f_{2} / 2}\left(\left(z-x_{1, n}+x_{2, n}-x_{3, n}\right) / 2\right) \\
x_{3, n+1}=\operatorname{prox}_{f_{3} / 2}\left(\left(z-x_{1, n}-x_{2, n}+x_{3, n}\right) / 2\right)
\end{array}\right.
$$

On the other hand, if $m=2$, (5.11) becomes

$$
\underset{x_{1} \in \mathscr{H}, x_{2} \in \mathscr{H}}{\operatorname{minimize}} f_{1}\left(x_{1}\right)+f_{2}\left(x_{2}\right)+\frac{1}{2}\left\|z-x_{1}-x_{2}\right\|^{2} .
$$


This problem is studied in [23], where an alternating algorithm is proposed which converges weakly to a solution to (5.14). In particular, if we take $f_{1}$ to be the indicator function of a nonempty closed convex set $C_{1} \subset \mathscr{H}$ and $f_{2}=\sigma_{C_{2}}$ to be the support function of a nonempty closed convex set $C_{2} \subset \mathscr{H}$, (5.14) becomes

$$
\underset{x_{1} \in C_{1}, x_{2} \in \mathscr{H}}{\operatorname{minimize}} \sigma_{C_{2}}\left(x_{2}\right)+\frac{1}{2}\left\|z-x_{1}-x_{2}\right\|^{2} .
$$

This problem is studied in [5].

The role of each potential $f_{i}$ in (5.11) is to promote certain known properties of the component $\bar{x}_{i}$. For instance, if some properties of the coefficients $\left(\left\langle\bar{x}_{i} \mid e_{i k}\right\rangle\right)_{k \in \mathbb{N}}$ of the decomposition of $\bar{x}_{i}$ in an orthonormal basis $\left(e_{i k}\right)_{k \in \mathbb{N}}$ of $\mathscr{H}$ are available, we can take (see $[16,20,25]$ for specific choices of the potentials $\left.\left(\phi_{i k}\right)_{k \in \mathbb{N}}\right)$

$$
\left.\left.f_{i}: \mathscr{H} \rightarrow\right]-\infty,+\infty\right]: x_{i} \mapsto \sum_{k \in \mathbb{N}} \phi_{i k}\left(\left\langle x_{i} \mid e_{i k}\right\rangle\right),
$$

where, for every $k \in \mathbb{N}, \phi_{i k} \in \Gamma_{0}(\mathbb{R})$ satisfies $\phi_{i k} \geq \phi_{i k}(0)=0$. If we adopt this model for each component in (5.11), we obtain

$$
\underset{x_{1} \in \mathscr{H}, \cdots, x_{m} \in \mathscr{H}}{\operatorname{minimize}} \sum_{i=1}^{m} \sum_{k \in \mathbb{N}} \phi_{i k}\left(\left\langle x_{i} \mid e_{i k}\right\rangle\right)+\frac{1}{2}\left\|z-\sum_{i=1}^{m} x_{i}\right\|^{2} .
$$

In addition, we derive from (5.16) and [23, Example 2.19] that (5.12) reduces to (we set $\lambda_{n} \equiv 1$ and $a_{i, n} \equiv 0$ for simplicity)

$$
x_{i, n+1}=\sum_{k \in \mathbb{N}} \operatorname{prox}_{\gamma_{n} \phi_{i k}}\left(\left\langle x_{i, n} \mid e_{i k}\right\rangle+\gamma_{n}\left(\left\langle z \mid e_{i k}\right\rangle-\sum_{j=1}^{m}\left\langle x_{j, n} \mid e_{i k}\right\rangle\right)\right) e_{i k} .
$$

\subsection{Signal synthesis}

Let $p=m(m-1) / 2$ be the cardinality of the set $\mathbb{K}=\left\{(i, j) \in\{1, \cdots, m\}^{2} \mid j>i\right\}$. For every $k=\left(k_{1}, k_{2}\right) \in \mathbb{K}$ set $z_{k}=0$ and

$$
\xi_{k i}= \begin{cases}1, & \text { if } i=k_{1} \\ -1, & \text { if } i=k_{2} \\ 0, & \text { otherwise }\end{cases}
$$

With this scenario, Problem 5.1 features pairwise quadratic couplings, which yields

$$
\underset{x_{1} \in \mathscr{H}, \cdots, x_{m} \in \mathscr{H}}{\operatorname{minimize}} \sum_{i=1}^{m} f_{i}\left(x_{i}\right)+\frac{1}{2} \sum_{i=1}^{m} \sum_{j=i+1}^{m}\left\|x_{i}-x_{j}\right\|^{2} .
$$

For instance, when $m=2$ and $f_{1}$ and $f_{2}$ are the indicator functions of nonempty closed convex sets $C_{1}$ and $C_{2}$ in $\mathscr{H}$, we obtain the classical problem

$$
\underset{x_{1} \in C_{1}, x_{2} \in C_{2}}{\operatorname{minimize}}\left\|x_{1}-x_{2}\right\|^{2}
$$


which has been studied in $[29,45]$. Another instance of (5.20) with $m=2$, is that obtained by taking $f_{2}: x \mapsto\|y-L x\|^{2} / 2$, where $L$ is a bounded linear operator from $\mathscr{H}$ to a real Hilbert space $\mathscr{G}$ and $y \in \mathscr{G}$. In this case (5.20) becomes

$$
\underset{x_{1} \in \mathscr{H}, x_{2} \in \mathscr{H}}{\operatorname{minimize}} f_{1}\left(x_{1}\right)+\frac{1}{2}\left\|y-L x_{2}\right\|^{2}+\frac{1}{2}\left\|x_{1}-x_{2}\right\|^{2} .
$$

This formulation arises in the image restoration problems of $[32,44]$ for specific choices of $f_{1}$ in finite dimensional spaces.

Since the matrix $\Delta=\Xi^{\top} \Xi=m \mathrm{Id}-1 \cdot 1^{\top}$ has largest eigenvalue $\lambda_{\max }=m$, we have $\beta_{3}=1 / m$ in Algorithm 5.1. In addition, (5.3) becomes

$$
x_{i, n+1}=x_{i, n}+\lambda_{n}\left(\operatorname{prox}_{\gamma_{n} f_{i}}\left(\left(1-m \gamma_{n}\right) x_{i, n}+\gamma_{n} \sum_{j=1}^{m} x_{j, n}\right)+a_{i, n}-x_{i, n}\right) .
$$

In particular, upon setting $\gamma_{n} \equiv 1 / m, \lambda_{n} \equiv 1$, and $a_{i, n} \equiv 0$, we obtain the parallel method

$$
\left\{\begin{aligned}
x_{1, n+1} & =\operatorname{prox}_{f_{1} / m}\left(\frac{1}{m} \sum_{j=1}^{m} x_{j, n}\right), \\
\vdots & \\
x_{m, n+1} & =\operatorname{prox}_{f_{m} / m}\left(\frac{1}{m} \sum_{j=1}^{m} x_{j, n}\right) .
\end{aligned}\right.
$$

\section{Application to multiframe signal representation}

This section is devoted to an application to multiframe signal processing in a real Hilbert space $\mathscr{G}$. Recall that a sequence $\left(e_{k}\right)_{k \in \mathbb{N}}$ in $\mathscr{G}$ is a frame if there exist constants $\mu$ and $v$ in $] 0,+\infty[$ such that $[24,30,38]$

$$
(\forall y \in \mathscr{G}) \quad \mu\|y\|^{2} \leq \sum_{k \in \mathbb{N}}\left|\left\langle y \mid e_{k}\right\rangle\right|^{2} \leq v\|y\|^{2} .
$$

The associated frame operator is the injective bounded linear operator

$$
F: \mathscr{G} \rightarrow \ell^{2}(\mathbb{N}): y \mapsto\left(\left\langle y \mid e_{k}\right\rangle\right)_{k \in \mathbb{N}}
$$

and its adjoint is the surjective bounded linear operator

$$
F^{*}: \ell^{2}(\mathbb{N}) \rightarrow \mathscr{G}:\left(\eta_{k}\right)_{k \in \mathbb{N}} \mapsto \sum_{k \in \mathbb{N}} \eta_{k} e_{k} .
$$

Frames extend the notion of orthonormal bases and they have been used in a number of variational signal processing problems due to their ability to efficiently capture a wide range signal features, e.g., $[12,16,26]$. We consider a variational formulation which exploits information on the frame representation of each signal component. In the case of $m=1$ component, a similar setting is considered in [16]. 
Problem 6.1. Set $\mathscr{H}=\ell^{2}(\mathbb{R})$ and let $\varphi \in \Gamma_{0}(\mathscr{G})$ be a $\tau$-Lipschitz differentiable function, for some $\tau \in] 0,+\infty\left[\right.$. For every $i \in\{1, \cdots, m\}$, let $\left(e_{i k}\right)_{k \in \mathbb{N}}$ be a frame of $\mathscr{G}$ with associated frame operator $F_{i}$ and, for every $k \in \mathbb{N}$, let $\phi_{i k} \in \Gamma_{0}(\mathbb{R})$ be such that $\phi_{i k} \geq \phi_{i k}(0)=0$. The problem is to

$$
\underset{\left(\eta_{1 k}\right)_{k \in \mathbb{N}} \in \mathscr{H}, \cdots,\left(\eta_{m k}\right)_{k \in \mathbb{N}} \in \mathscr{H}}{\operatorname{minimize}} \sum_{i=1}^{m} \sum_{k \in \mathbb{N}} \phi_{i k}\left(\eta_{i k}\right)+\varphi\left(\sum_{i=1}^{m} \sum_{k \in \mathbb{N}} \eta_{i k} e_{i k}\right),
$$

under the assumption that solutions exist.

Algorithm 6.1. Set $\mathscr{H}=\ell^{2}(\mathbb{R})$ and

$$
\beta_{4}=\frac{1}{\tau \sum_{i=1}^{m} v_{i}}
$$

where, for every $\left.i \in\{1, \cdots, m\}, v_{i} \in\right] 0,+\infty\left[\right.$ is the upper frame constant of $\left(e_{i k}\right)_{k \in \mathbb{N}}$ (see (6.1)). Fix $\varepsilon$ in $] 0, \min \left\{1, \beta_{4}\right\}\left[,\left(\lambda_{n}\right)_{n \in \mathbb{N}}\right.$ in $[\varepsilon, 1],\left(\gamma_{n}\right)_{n \in \mathbb{N}}$ in $\left[\varepsilon, 2 \beta_{4}-\varepsilon\right]$, and let $\left(\eta_{1 k, 0}\right)_{k \in \mathbb{N}}, \cdots,\left(\eta_{m k, 0}\right)_{k \in \mathbb{N}}$ be sequences in $\mathscr{H}$. For every $i \in\{1, \cdots, m\}$ set, for every $n \in \mathbb{N}$,

$$
\begin{aligned}
(\forall k \in \mathbb{N}) \quad \eta_{i k, n+1}= & \eta_{i k, n}+\lambda_{n}\left(\operatorname { p r o x } _ { \gamma _ { n } \phi _ { i k } } \left(\eta_{i k, n}-\gamma_{n}\left(\left\langle\nabla \varphi\left(\sum_{j=1}^{m} \sum_{k \in \mathbb{N}} \eta_{j k, n} e_{j k}\right) \mid e_{i k}\right\rangle\right.\right.\right. \\
& \left.\left.\left.+\beta_{i k, n}\right)\right)+\alpha_{i k, n}-\eta_{i k, n}\right),
\end{aligned}
$$

where $\left(\alpha_{i k, n}\right)_{(k, n) \in \mathbb{N}^{2}}$ and $\left(\beta_{i k, n}\right)_{(k, n) \in \mathbb{N}^{2}}$ are real sequences such that

$$
\sum_{n \in \mathbb{N}} \sqrt{\sum_{k \in \mathbb{N}}\left|\alpha_{i k, n}\right|^{2}}<+\infty \text { and } \sum_{n \in \mathbb{N}} \sqrt{\sum_{k \in \mathbb{N}}\left|\beta_{i k, n}\right|^{2}}<+\infty .
$$

Remark 6.1. In some cases, it may be possible to obtain a sharper bound than (6.5); see [16, Remark 5.3].

Corollary 6.1. Let $\left(\left(\eta_{1 k, n}\right)_{k \in \mathbb{N}}\right)_{n \in \mathbb{N}}, \cdots,\left(\left(\eta_{m k, n}\right)_{k \in \mathbb{N}}\right)_{n \in \mathbb{N}}$ be sequences generated by Algorithm 6.1. Then, for every $i \in\{1, \cdots, m\}$ and $k \in \mathbb{N},\left(\eta_{i k, n}\right)_{n \in \mathbb{N}}$ converges to a point $\eta_{i k} \in \mathbb{R}$, and $\left(\left(\eta_{i k}\right)_{k \in \mathbb{N}}\right)_{1 \leq i \leq m}$ is a solution to Problem 6.1.

Proof. Problem 6.1 is a particular case of Problem 1.1 in which $p=1, \varphi_{1}=\varphi$, and for every $\left.\left.i \in\{1, \cdots, m\}, \mathscr{H}_{i}=\mathscr{H}=\ell^{2}(\mathbb{R}), f_{i}: \mathscr{H} \rightarrow\right]-\infty,+\infty\right]:\left(\eta_{k}\right)_{k \in \mathbb{N}} \mapsto \sum_{k \in \mathbb{N}} \phi_{i k}\left(\eta_{k}\right)$, and $L_{1 i}=F_{i}^{*}$. In addition, we derive from (6.5), (1.4), and (6.1) that $\beta_{4} \leq \beta_{1}$. Finally, using [23, Example 2.19], we deduce that Algorithm 6.1 is a particular case of Algorithm 1.1. The result therefore follows from Theorem 1.2.

We conclude with a specific instance of Problem 6.1. 
Example 6.1. Let $\mathscr{K}$ be a real Hilbert space, let $z \in \mathscr{K}$, and let $L: \mathscr{G} \rightarrow \mathscr{K}$ be linear and bounded. Set $\varphi=\|z-L \cdot\|^{2} / 2$ and, for every $i \in\{1, \cdots, m\}$ and $k \in \mathbb{N}$, set $\phi_{i k}=w_{i k}|\cdot| p_{i}$, where $p_{i} \in[1,2]$ and $\left.w_{i k} \in\right] 0,+\infty[$. Then (6.4) becomes

$$
\underset{\left(\eta_{1 k}\right)_{k \in \mathbb{N}} \in \mathscr{H}, \cdots,\left(\eta_{m k}\right)_{k \in \mathbb{N}} \in \mathscr{H}}{\operatorname{minimize}} \sum_{i=1}^{m} \sum_{k \in \mathbb{N}} w_{i k}\left|\eta_{i k}\right|^{p_{i}}+\frac{1}{2}\left\|z-L\left(\sum_{i=1}^{m} \sum_{k \in \mathbb{N}} \eta_{i k} e_{i k}\right)\right\|^{2} .
$$

This problem is studied in [37].

Acknowledgments This work was supported by the Agence Nationale de la Recherche under grant ANR-08-BLAN-0294-02.

\section{References}

[1] F. Acker And M. A. Prestel, Convergence d'un schéma de minimisation alternée, Ann. Fac. Sci. Toulouse V. Sér. Math., 2 (1980), pp. 1-9.

[2] S. Anthoine, E. Pierpaoli, And I. Daubechies, Deux méthodes de déconvolution et séparation simultanées; application à la reconstruction des amas de galaxies, Traitement Signal, 23 (2006), pp. 439-447.

[3] H. Attouch, J. Bolte, P. Redont, and A. Soubeyran, Alternating proximal algorithms for weakly coupled convex minimization problems. Applications to dynamical games and PDE's, J. Convex Anal., 15 (2008), pp. 485-506.

[4] H. Attouch, L. M. Briceño-Arias, And P. L. Combettes, A parallel splitting method for coupled monotone inclusions, http://www.ann.jussieu.fr/ plc/abc123.pdf.

[5] J.-F. Aujol, G. Aubert, L. Blanc-Féraud, and A. Chambolle, Image decomposition into a bounded variation component and an oscillating component, J. Math. Imaging Vision, 22 (2005), pp. 71-88.

[6] J.-F. Aujol And A. Chambolle, Dual norms and image decomposition models, Int. J. Comput. Vision, 63 (2005), pp. 85-104.

[7] J.-F. Aujol, G. Gilboa, T. Chan, And S. Osher, Structure-texture image decomposition - Modeling, algorithms, and parameter selection, Int. J. Comput. Vision, 67 (2006), pp. 111-136.

[8] J.-F. Aujol And S. H. KANG, Color image decomposition and restoration, J. Vis. Commun. Image Represent., 17 (2006), pp. 916-928.

[9] J.-B. BAIllon AND G. HADDAD, Quelques propriétés des opérateurs angle-bornés et ncycliquement monotones, Israel J. Math., 26 (1977), pp. 137-150.

[10] H. H. BauschKe, P. L. Combettes, AND D. Nold, Joint minimization with alternating Bregman proximity operators, Pacific J. Optim., 2 (2006), pp. 401-424.

[11] C. L. Byrne, Signal Processing - A Mathematical Approach, A. K. Peters, Wellesley, MA, 2005.

[12] J.-F. CAI, R. H. Chan, L. Shen, AND Z. Shen, Convergence analysis of tight framelet approach for missing data recovery, Adv. Comput. Math., 31 (2009), pp. 87-113.

[13] Y. CENSOR AND T. ElfVIng, A multiprojection algorithm using Bregman projections in a product space, Numer. Algorithms, 8 (1994), pp. 221-239.

[14] Y. Censor and S. A. Zenios, Parallel Optimization: Theory, Algorithms and Applications, Oxford University Press, New York, 1997.

[15] T. F. ChAN, S. EsEDoglu, AND F. E. PARK, Image decomposition combining staircase reduction and texture extraction, J. Vis. Commun. Image Represent., 18 (2007), pp. 464-486. 
[16] C. Chaux, P. L. Combettes, J.-C. Pesquet, And V. R. WAJs, A variational formulation for framebased inverse problems, Inverse Problems, 23 (2007), pp. 1495-1518.

[17] C. Combari, M. Laghdir, AND L. Thibault, A note on subdifferentials of convex composite functionals, Arch. Math. (Basel), 67 (1996), pp. 239-252.

[18] P. L. Combettes AND P. Bondon, Hard-constrained inconsistent signal feasibility problems, IEEE Trans. Signal Process., 47 (1999), pp. 2460-2468.

[19] P. L. Combettes And J.-C. Pesquet, A Douglas-Rachford splitting approach to nonsmooth convex variational signal recovery, IEEE J. Selected Topics Signal Process., 1 (2007), pp. 564-574.

[20] P. L. Combettes And J.-C. Pesquet, Proximal thresholding algorithm for minimization over orthonormal bases, SIAM J. Optim., 18 (2007), pp. 1351-1376.

[21] P. L. Combettes AND J.-C. PesQuet, A proximal decomposition method for solving convex variational inverse problems, Inverse Problems, 24 (2008), Article ID 065014.

[22] P. L. Combettes And H. J. TRussell, The use of the noise properties in set theoretic estimation, IEEE Trans. Signal Process., 39 (1991), pp. 1630-1641.

[23] P. L. Combettes AND V. R. WAJs, Signal recovery by proximal forward-backward splitting, Multiscale Model. Simul., 4 (2005), pp. 1168-1200.

[24] I. Daubechies, Ten Lectures on Wavelets, SIAM, Philadelphia, PA, 1992.

[25] I. Daubechies, M. Defrise, and C. De Mol, An iterative thresholding algorithm for linear inverse problems with a sparsity constraint, Comm. Pure Appl. Math., 57 (2004), pp. 14131457.

[26] S. Durand and M. Nikolova, Denoising of frame coefficients using $\ell^{1}$ data-fidelity term and edge-preserving regularization, Multiscale Model. Simul., 6 (2007), pp. 547-576.

[27] J. Gilles, Noisy image decomposition: a new structure, texture and noise model based on local adaptivity, J. Math. Imaging Vision, 28 (2007), pp. 285-295.

[28] R. Glowinski, J.-L. Lions, And R. Trémolières, Analyse Numérique des Inéquations Variationnelles. Dunod, Paris, 1976.

[29] M. Goldburg and R. J. Marks II, Signal synthesis in the presence of an inconsistent set of constraints, IEEE Trans. Circuits and Systems, 32 (1985), pp. 647-663.

[30] C. E. Heil and D. F. Walnut, Continuous and discrete wavelet transforms, SIAM Rev., 31 (1989), pp. 628-666.

[31] J.-B. Hiriart-Urruty and C. Lemaréchal, Convex Analysis and Minimization Algorithms, Springer-Verlag, New York, 1993.

[32] Y. HuANG, M. K. NG, AND Y.-W. WEN, A fast total variation minimization method for image restoration, Multiscale Model. Simul., 7 (2008), pp. 774-795.

[33] M. KANG, Generalized multichannel image deconvolution approach and its applications, Opt. Eng., 37 (1998), pp. 2953-2964.

[34] A. Katsaggelos, K. LAY, AND N. Galatsanos, A general framework for frequency domain multichannel signal processing, IEEE Trans. Image Process., 2 (1993), pp. 417-420.

[35] J.-J. Moreau, Proximité et dualité dans un espace Hilbertien, Bull. Soc. Math. France, 93 (1965), pp. 273-299.

[36] R. T. Rockafellar, Convex Analysis, Princeton University Press, Princeton, NJ, 1970.

[37] G. TeschKe, Multi-frame representations in linear inverse problems with mixed multiconstraints, Appl. Comput. Harmon. Anal., 22 (2007), pp. 43-60.

[38] R. Tolimieri AND M. AN, Time-Frequency Representations, Birkhäuser, Boston, MA, 1998.

[39] H. J. Trussell and M. R. Civanlar, The feasible solution in signal restoration, IEEE Trans. Acoust., Speech, Signal Process., 32 (1984), pp. 201-212.

[40] D. Tschumperlé AND R. Deriche, Diffusion PDEs on vector-valued images, Signal Process. Mag., 19 (2002), pp. 16-25. 
[41] L. A. Vese And S. J. Osher, Modeling textures with total variation minimization and oscillating patterns in image processing, J. Sci. Comput., 19 (2003), pp. 553-572.

[42] L. A. Vese AND S. J. Osher, Image denoising and decomposition with total variation minimization and oscillatory functions, J. Math. Imaging Vision, 20 (2004), pp. 7-18.

[43] Y. WANG, J. YANG, W. YIN, AND Y. ZHANG, A new alternating minimization algorithm for total variation image reconstruction, SIAM J. Imaging Sci., 1 (2008), pp. 248-272.

[44] Y.-W. Wen, M. K. NG, W.-K. ChIng, Iterative algorithms based on decoupling of deblurring and denoising for image restoration, SIAM J. Sci. Comput., 30 (2008), pp. 2655-2674.

[45] D. C. Youla AND V. Velasco, Extensions of a result on the synthesis of signals in the presence of inconsistent constraints, IEEE Trans. Circuits and Systems, 33 (1986), pp. 465-468.

[46] C. ZĂLInEscu, Convex Analysis in General Vector Spaces, World Scientific, River Edge, NJ, 2002. 